\title{
On the Effects of Complex Conjugate Medium on TM Scattering by a Strip
}

\author{
Amjad Imran ${ }^{1}$, Ahsan Illahi' \\ ${ }^{1}$ National Center for Physics (NCP), Quaid-i-Azam University, Islamabad, Pakistan; ${ }^{2}$ Department of Physics, Allama Iqbal Open \\ University, Islamabad, Pakistan. \\ Email: amjadqau@hotmail.com, ahsanilahi@gmail.com
}

Received April 24th, 2011; revised May 27 $7^{\text {th }}, 2011$; accepted June $10^{\text {th }}, 2011$.

\begin{abstract}
In this paper, Method of Kobayashi Potential is used to determine the scattering behavior of a strip which is placed at the air-complex conjugate medium interface. And discussion is presented that how the complex conjugate medium modifies the scattering properties of the strip. A comparison is also given with that if we replace the conjugate medium with standard dielectric medium. E-polarized electromagnetic plane wave is supposed to be obliquely incident upon the geometry. Scattered fields in both the half spaces are supposed in terms of unknown weighting functions. Discontinuous properties of Weber-Schafheitlin integral and orthogonal properties of Jacobi's polynomials are used to determine these unknown weighting functions. Far scattered fields have been calculated using Saddle Point Method and computed for different parameters of interest.
\end{abstract}

Keywords: Complex Conjugate Medium, Kobayashi Potential Method, Scattering from Strip, Mixed Boundary Value Problem Etc

\section{Introduction}

The complex value of the constitutive parameters (i.e. permittivity and permeability) of a medium cause the medium to be lossy as the refractive index $\eta=\sqrt{\varepsilon_{r} \mu_{r}}$ or the propagation constant of the medium also become complex (where $\varepsilon_{r}$ and $\mu_{r}$ is the relative permittivity and relative permeability of the medium). But such medium may also be lossless if $\varepsilon_{r}=\mu_{r}^{*}$ (that is they are complex conjugate of each other) because in this case refractive index/propagation constant will become real and this real value of $\eta$ will ensure the unattanuated wave propagation through the medium. Such medium is named as complex conjugate medium by Dregoman in his recently published paper [1]. In this paper, he has discussed the reflection and transmission properties of this medium. He also has proposed a scheme to realize such materials. These materials have many potential applications in optoelectronics, miniaturized amplifiers and lasers.

In the present work, analysis has been presented which investigates the effects of complex valued constitutive parameters of the complex conjugate medium on the scattering properties of a conducting strip. Illustrative computations are given for far field patterns as the func- tion of imaginary part of permittivity/permeability whose value is zero for standard dielectric medium. Present analysis is based on the Kobayashi Potential (KP) Method. This method has successfully been applied to various problems in electromagnetic and acoustics [2-6]. The method exploits the discontinuous properties of Weber-Schafheitlin's integrals and orthogonal properties of Jacobi's polynomials to construct the solution. In this method the problem at its final stage reduce to matrix equations. The elements of matrix equations are usually infinite integrals which has branch points as well as poles depending upon the nature of the problem. Analytic solutions of these integrals are not possible for most of the cases. Therefore algorithms are developed to numerically solve these integrals and hence the matrix equations.

\section{Formulation and Solution of the Problem}

The geometry of the problem and coordinate system are shown in Figure 1. A thin conducting strip of width $2 \alpha$ is located at the interface between air and complex conjugate medium. $\left(\varepsilon_{0}, \mu_{0}\right)$ are supposed to be the constitutive parameters of upper half space $(y>0)$ medium, while the lower half space $(y<0)$, which is occupied by the complex conjugate medium, has the constitutive 


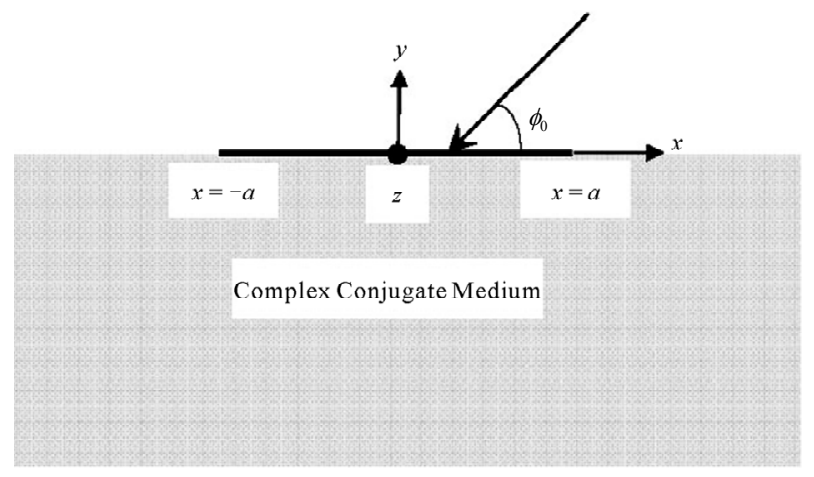

Figure 1. Geometry of the problem.

parameters $(\varepsilon, \mu)$. For the sake of simplicity, we suppose that an E-polarized field, $E_{z}^{i}$, is obliquely incident upon the geometry, therefore

$$
E_{z}^{i}=\exp \left[j k_{0}\left(x \cos \phi_{0}+y \sin \phi_{0}\right)\right]
$$

where $\phi_{0}$ is the angle of incidence with $\mathrm{x}$-axis and $k_{0}$ be the wave number for $y>0$ half space. In each region, scattered field may be assumed in terms of unknown weighting functions. Therefore, expressions for scattered field, $E_{z}^{d+}$ in upper half space and in lower half space, can be assumed in the form

$$
\begin{aligned}
E_{Z}^{d^{+}}= & \int_{0}^{\infty}\left[g_{1}(\xi) \cos \left(\xi x_{a}\right)+g_{2}(\xi) \sin \left(\xi x_{a}\right)\right] \\
& \cdot \exp \left[-\sqrt{\xi^{2}-\kappa_{0}^{2}}\right] \mathrm{d} \xi \quad y>0 \\
E_{Z}^{d^{-}}= & \int_{0}^{\infty}\left[h_{1}(\xi) \cos \left(\xi x_{a}\right)+h_{2}(\xi) \sin \left(\xi x_{a}\right)\right] \\
& \cdot \exp \left[\sqrt{\xi^{2}-\kappa^{2}}\right] \mathrm{d} \xi \quad y<0
\end{aligned}
$$

where $k=\omega \sqrt{\mu \varepsilon}$ is the propagation constant of complex conjugate medium and $\kappa_{0}=k_{0} a, \kappa=k a, x_{a}=\frac{x}{a}$,

$y_{a}=\frac{y}{a}$ and the functions $g_{1,2}(\xi), h_{1,2}(\xi)$ are the weighting functions to be determined from the boundary conditions.

Geometry supports the following boundary conditions

$$
\begin{aligned}
& \text { 1) }\left.\quad E_{z}^{t}\right|_{y=0}=0 \quad \text { for }\left|x_{a}\right| \leq 1 \\
& \text { 2) }\left.\quad E_{z}^{t}\right|_{y=0_{+}}=\left.E_{z}^{t}\right|_{y=0_{-}} \quad \text { for all } x_{a} \\
& \text { 3) }\left.H_{z}^{t}\right|_{y=0_{+}}=\left.H_{z}^{t}\right|_{y=0_{-}} \quad \text { for }\left|x_{a}\right| \geq 1
\end{aligned}
$$

where superscript stands for total. Applying boundary condition 2) we have

$$
g_{1}(\xi)=h_{1}(\xi) ; g_{2}(\xi)=h_{2}(\xi)
$$

Imposing the boundary conditions 1) and 3) give

$$
\begin{aligned}
& \int_{0}^{\infty}\left[\sqrt{\xi^{2}-\kappa^{2}}+\mu_{r} \sqrt{\xi^{2}-\kappa_{0}^{2}}\right] \\
& \cdot\left[g_{1}(\xi) \cos \left(\xi x_{a}\right)+g_{2}(\xi) \sin \left(\xi x_{a}\right)\right] \mathrm{d} \xi=0 \\
& \int_{0}^{\infty}\left[g_{1}(\xi) \cos \left(\xi x_{a}\right)+g_{2}(\xi) \sin \left(\xi x_{a}\right)\right] \mathrm{d} \xi \\
& =(1+R) \exp \left(j \kappa_{0} x_{a} \cos \phi_{0}\right)
\end{aligned}
$$

where $R$ is the reflection coefficient of the complex conjugate half space without strip and

$$
R=\frac{Z_{t} \sin \phi_{0}-Z_{0} \sin \phi_{t}}{Z_{t} \sin \phi_{0}+Z_{0} \sin \phi_{t}} ; \quad k_{t} \cos \phi_{t}=k_{0} \cos \phi_{0}
$$

where $Z_{0}$ and $Z_{t}$ are intrinsic impedances of free space and complex conjugate medium respectively and $\phi_{t}$ is the angle of refraction which is related to angle of incidence through second relation of last expression.

Dependency of $g_{1}(\xi)$ and $g_{2}(\xi)$ may be decided by comparing (3a) with Weber-Schafheitlin's integral and exploiting the properties of Gamma function at negative integer values, we have

$$
\begin{aligned}
& g_{1}(\xi)=\sum_{m=0}^{\infty} A_{m} \frac{J_{2 m}(\xi)}{\sqrt{\xi^{2}-\kappa^{2}}+\mu_{r} \sqrt{\xi^{2}-\kappa_{0}^{2}}} \\
& g_{2}(\xi)=\sum_{m=0}^{\infty} B_{m} \frac{J_{2 m+1}(\xi)}{\sqrt{\xi^{2}-\kappa^{2}}+\mu_{r} \sqrt{\xi^{2}-\kappa_{0}^{2}}}
\end{aligned}
$$

where $A_{m}$ and $B_{m}$ are expansion coefficients and $J_{m}($.$) be the Bessel's function of order m$. Separating even and odd functions of the expression (3b) and putting the values of weighting functions as given in (4), we get

$$
\begin{aligned}
& \sum_{m=0}^{\infty} A_{m} \int_{0}^{\infty} \frac{J_{2 m}(\xi) \cos \left(\xi x_{a}\right)}{\sqrt{\xi^{2}-\kappa^{2}}+\mu_{r} \sqrt{\xi^{2}-\kappa_{0}^{2}}} \mathrm{~d} \xi \\
& =-\frac{2 Z_{t} \sin \phi_{0}}{Z_{t} \sin \phi_{0}+Z_{0} \sin \phi_{t}}\left(\cos \kappa_{0} x_{a} \phi_{0}\right) \\
& \sum_{m=0}^{\infty} B_{m} \int_{0}^{\infty} \frac{J_{2 m+1}(\xi) \sin \left(\xi x_{a}\right)}{\sqrt{\xi^{2}-\kappa^{2}}+\mu_{r} \sqrt{\xi^{2}-\kappa_{0}^{2}}} \mathrm{~d} \xi \\
& =-\frac{2 j Z_{t} \sin \phi_{0}}{Z_{t} \sin \phi_{0}+Z_{0} \sin \phi_{t}}\left(\sin \kappa_{0} x_{a} \phi_{0}\right)
\end{aligned}
$$

Expanding the trigonometric functions in above expressions in terms of Jacobi's polynomials $v_{n}^{ \pm \frac{1}{2}}\left(x^{2}\right)$ [7], we get the following matrix equations 


$$
\begin{gathered}
\sum_{m=0}^{\infty}[G(2 m, 2 n)]\left[A_{m}\right]=\vartheta\left[J_{2 n}\left(\kappa_{0} \cos \phi_{0}\right)\right] \\
\sum_{m=0}^{\infty}[G(2 m+1,2 n+1)]\left[B_{m}\right]=j \vartheta\left[J_{2 n+1}\left(\kappa_{0} \cos \phi_{0}\right)\right] \\
n=0,1,2, \cdots
\end{gathered}
$$

where notations used in above expressions are defined as

$$
\begin{gathered}
\vartheta=\frac{2 Z_{t} \sin \phi_{0}}{Z_{t} \sin \phi_{0}+Z_{0} \sin \phi_{t}} \\
G(\mu, \nu)=\int_{0}^{\infty} \frac{J_{\mu}(\xi) J_{\nu}(\xi)}{\sqrt{\xi^{2}-\kappa^{2}}+\mu_{r} \sqrt{\xi^{2}-\kappa_{0}^{2}}} \mathrm{~d} \xi
\end{gathered}
$$

In obtaining (6a) and (6b) we have made use of following relations

$$
\begin{aligned}
& \cos x=J_{\frac{1}{2}}(x) ; \sin x=J_{-\frac{1}{2}}(x) \\
& x^{-m / 2} J_{m}(\xi \sqrt{x}) \\
& =\sum_{m=0}^{\infty} \frac{\sqrt{8}(2 n+m+3 / 2) \Gamma(n+m+3 / 2)}{\Gamma(n+1) \Gamma(m+1)} \frac{J_{2 n+m+3 / 2}(\xi)}{\xi^{\frac{3}{2}}} v_{n}^{m}
\end{aligned}
$$

and orthogonal properties of Jacobi's polynomials $v_{n}^{m}(x)$.

\section{Far Scattered Field}

Far scattered fields can be calculated by using Saddle Point Method. Therefore, substituting the values of $g_{1}(\xi)$ and $g_{2}(\xi)$ and then applying the said method, (1b) finally reduces to

$$
\begin{aligned}
E_{z}^{d^{+}}= & C(k \rho) \frac{\sin \phi}{\mu_{r} \sin \phi+\sqrt{\eta^{2}-\cos ^{2} \phi}} \sum_{m=0}^{\infty} A_{m} J_{2 m}\left(\kappa_{0} \cos \phi\right) \\
& +j B_{m} J_{2 m+1}\left(\kappa_{0} \cos \phi\right)
\end{aligned}
$$

where $C(k \rho)=\sqrt{\frac{2}{\pi \kappa \rho}} \exp \left(-j k \rho-j \frac{\pi}{4}\right), \phi$ is the angle of observation and $\eta=\frac{\kappa}{\kappa_{0}}$ is the refractive index of lower half space. Expansion coefficients $A_{m}$ and $B_{m}$ can be computed using matrix Equations (6a) and (6b).

\section{Results and Discussion}

Since both $\varepsilon_{r}$ and $\mu_{r}$ are complex quantities, so we can consider $\varepsilon_{r}=a+j b$ or in general $\varepsilon_{r}=m(a+j b)$ such that $\mu_{r}=a-j b$ and refractive index of complex conjugate medium $\eta=\sqrt{m\left(a^{2}+b^{2}\right)}$. Equation (6a) and (6b) are the matrix equations which can be used to determine the values of unknown expansion coefficients $A_{m}$ and $B_{m} . G(m, n)$ are the matrices, the elements of which are in terms of infinite integrals as indicated by (6d). Algorithms were developed to numerically evaluate these integrals. The matrix size was taken as

$m \times n=\left(2 \kappa_{0}+1\right) \times\left(2 \kappa_{0}+1\right)$. The values of expansion coefficients $A_{m}$ and $B_{m}$, as determined from (6a) and (6b), were then used in Equation (7) to determine the far scattered fields in upper half space. Since the most important parameter in our discussion is the imaginary part of permittivity/permeability that is designated by $b$. Therefore, Figure 2 represents the dependence of real part of scattered field on $b$. This figure also gives the comparison if we replace complex conjugate medium in lower half space with standard dielectric medium. For the standard dielectric case we have taken $\mu_{r}=1.0$ and $\eta=\sqrt{m\left(a^{2}+b^{2}\right)}$. Figure 3 gives imaginary part of the scattered field from complex conjugate medium and standard dielectric medium. These figures show that the real and imaginary parts of scattered field follow the similar trend for the case of dielectric medium. They only differ quantitatively. But for the case of complex conjugate medium, the situation is different. The behaviors of these parts of the scattered field with respect to $b$ are distinct. This situation may be exploited to differentiate complex conjugate materials from standard dielectric materials of same refractive index [8,9]. Figure 4 gives the variations in the far scattered field as a function of $m$, for $a=2.0, \kappa_{0}=4.0, \phi=\phi_{0}=\frac{\pi}{2}$. This figure shows that as we increase the values of $m$, the strength of the fields decrease. Figure 5 shows the influence of $b$ on field distribution in whole upper half space.

\section{Conclusions}

Complex conjugate and ordinary dielectric materials are

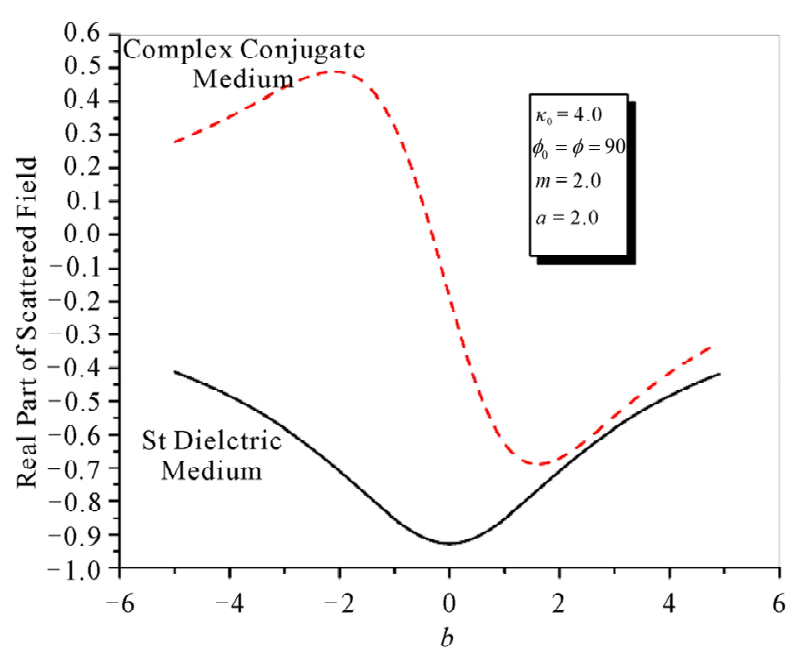

Figure 2. Dependence of real part of scattered field on $b$. 


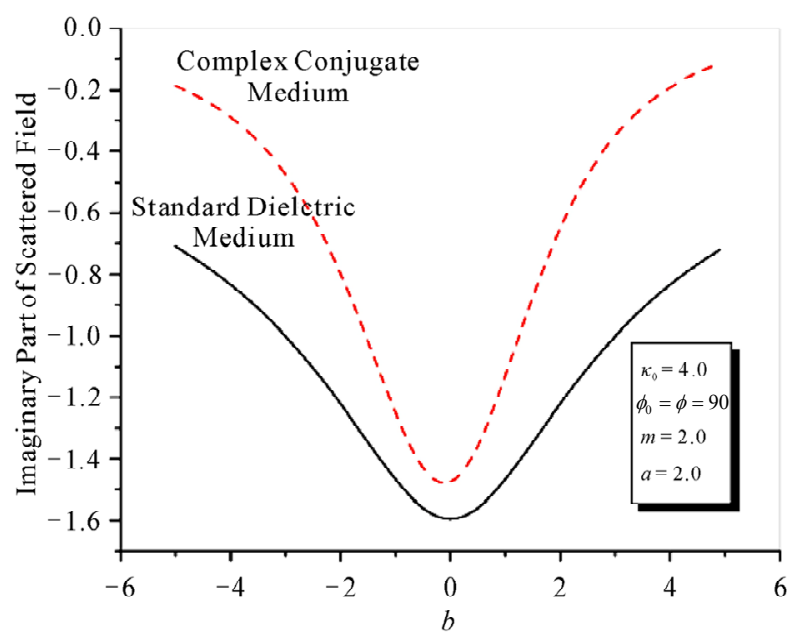

Figure 3. Dependence of imagnary part of scattered field on b.

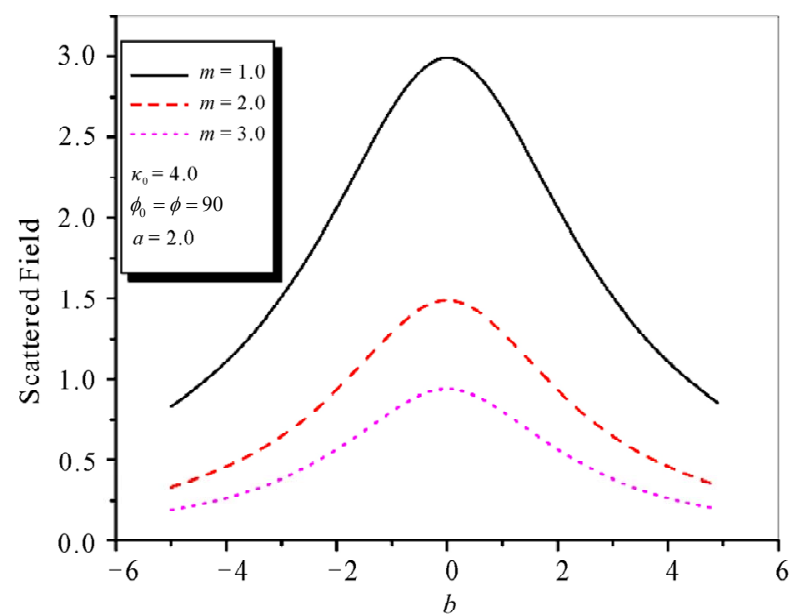

Figure 4. Effects of $\mathrm{m}$ on scattered field from complex conjugate medium.

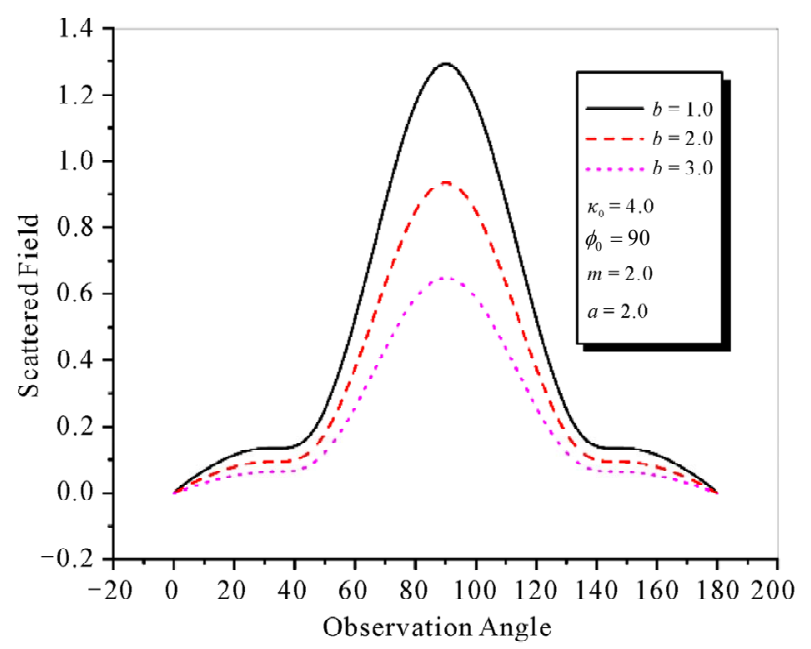

Figure 5. Scattered field in upper half plane. both lossless but distinction between then is that the constitutive parameters of complex conjugate materials are complex valued and that of ordinary materials are real valued of same sign. In the present study, the effects of these newly conceived complex conjugate materials on the scattering properties of a strip are analyzed. For this purpose, the Method of Kobayashi Potential is used which is well known in dealing with mixed boundary problems. A comparison with ordinary dielectric material with same refractive index is also presented. It is concluded that behavior of real and imaginary parts of the scattered field for both the cases differ qualitatively and quantitatively. Although both are lossless media but still they are distinguishable.

\section{REFERENCES}

[1] D. Dragoman, "Complex Conjugate Media: Alternative Configurations for Miniaturized Lasers," Optics Communication, Vol. 284, No. 8, 2011, pp. 2095-2098. doi:10.1016/j.optcom.2010.12.069

[2] I. Kobayashi, "Collected Papers of I. Kobayashi," Memorial Publication Committee, Faculty of Engineering, Nihon University, Tokyo, 1970

[3] K. Hongo, "Diffraction of Electromagnetic Plane Wave by the Slit," Trans. Inst, Elect. and Comm. Engrg, of Japan, Vol. 55-B, No. 6, 1972, pp. 328-330.

[4] K. Hongo, "Diffraction of Electromagnetic Plane Wave by an Infinite Slit Embedded in an Anisotropic Plasma," Journal of Applied Physics, Vol. 43, No. 12, 1972, pp. 4996-5001. doi:10.1063/1.1661059

[5] K. Hongo, "Diffraction by a Flanged Parallel Plate Waveguide," Radio Science, Vol. 7, No. 10, 1972, pp. 955-963. doi:10.1029/RS007i010p00955

[6] K. Hongo and H. Serizawa, "Diffraction of an Acoustic plane Wave by a Rectangular Plate," Journal of Applied Physics, Vol. 82, No. 6, 1997, pp. 2719-2729.

[7] M. Abramowitz and I. A. Stegun, "Handbook of Mathematical Functions," Dover Publishing Inc. New York, 1968.

[8] H. Fujiwara, "Spectroscopic Ellipsometry: Principles and Applications," Wiley, Hoboken, 2007.

[9] H. G. Tompkins and W. A. McGahan, "Spectroscopic Ellipsometry and Reflectometry: A User's Guide," Wiley Interscience, 1999. doi:10.1063/1.366266 